\title{
Novel self-nanomicellizing solid dispersion based on rebaudioside A: a potential nanoplatform for oral delivery of curcumin
}

This article was published in the following Dove Medical Press journal: International Journal of Nanomedicine

\author{
Yuzhen Hou' \\ Hui Wang' \\ Fan Zhang' \\ Fengyuan Sun' \\ Meng Xin ${ }^{1,2}$ \\ Mengshuang $\mathrm{Li}^{1,3}$ \\ Jun $\mathrm{Li}^{4}$ \\ Xianggen $\mathrm{Wu}^{\prime}$ \\ 'Department of Pharmacy, College \\ of Chemical Engineering, Qingdao \\ University of Science and Technology, \\ Qingdao 266042, China; ${ }^{2}$ Department \\ of Ophthalmology, Yantai Affiliated \\ Hospital of Binzhou Medical \\ University, Yantai 264100, China; \\ ${ }^{3}$ Pharmacy Intravenous Admixture \\ Services, Qingdao Women and \\ Children's Hospital, Qingdao 266034, \\ China; ${ }^{4}$ Qingdao Eye Hospital, \\ Shandong Eye Institute, Shandong \\ Academy of Medical Sciences, \\ Qingdao 26607I, China
}

Correspondence: Xianggen Wu Department of Pharmacy, College of Chemical Engineering, Qingdao University of Science and Technology, 53 Zhengzhou Road, Shibei District, Qingdao 266042, China $\mathrm{Tel} / \mathrm{fax}+8653284023003$ Email wuxianggen@I26.com
Purpose: Rebaudioside A (RA) has nanocarrier characteristics that allow it to self-assemble into micelles in aqueous solutions. The purpose of this study was to determine if a self-nanomicellizing solid dispersion based on RA could be utilized as an oral nano-drug delivery system.

Materials and methods: Curcumin (Cur) served as a model hydrophobic drug, and a Curloaded self-nanomicellizing solid dispersion based on RA (RA-Cur) was formulated. The properties of RA-Cur in the solid state and in aqueous solution were characterized. The antioxidant activity and mechanism of RA-Cur endocytosis were also investigated. The pharmacokinetics, biodistribution in the intestinal tract, and anti-inflammation properties were also evaluated in vivo. Results: RA-Cur could be easily fabricated, and it self-assembled into ultrasmall micelles (particle size $\sim 4 \mathrm{~nm}$ ) in a homogeneous distribution state (polydispersity index $<0.2$ ) when dissolved in water. Cur was readily encapsulated into RA micelles and this improved its water solubility (to $14.34 \pm 1.66 \mathrm{mg} / \mathrm{mL}$ ), as well as its in vitro release and membrane permeability. The antioxidant activities of Cur in RA-Cur were also significantly improved. Biodistribution in the intestinal tract confirmed a significant enhancement of Cur absorption in the duodenum, jejunum, and ileum by encapsulation in RA-Cur, and the absorption of RA-Cur was governed by mixed transcytosis mechanisms. Pharmacokinetic tests of RA-Cur in rats revealed a dramatic 19.06-fold enhancement of oral bioavailability when compared to free Cur. More importantly, oral administration of RA-Cur could efficiently ameliorate ulcerative colitis in a mouse model induced by dextran sodium sulfate. Conclusion: Self-nanomicellizing solid dispersions based on RA have great potential as novel oral nano-drug delivery systems for hydrophobic drugs such as Cur.

Keywords: rebaudioside A, curcumin, self-nanomicellizing, micelle, solid dispersion, drug delivery

\section{Introduction}

Rebaudioside A (RA) is a widely used sweetener obtained from the plant Stevia rebaudiana Bertoni. However, the applications of RA extend beyond its use as a sugar substitute, taste modifier, and sweetening agent as it also has biological activities that include antihyperlipidemic, anti-lipid peroxidative, and antioxidant properties. ${ }^{1}$ These features make RA potentially useful in the pharmaceutical and nutriological fields.

RA is a steviol glycoside - a diterpene with a steviol backbone and mono- and trisaccharide carbohydrate residues at the $\mathrm{C} 13$ and C19 positions (Figure S1). ${ }^{2}$ This combination of both hydrophobic diterpene and hydrophilic sugar side chain(s) in its structure allows RA to form micelles in aqueous solutions. Therefore, RA is of considerable clinical interest due to its potential for use as a nanotechnological drug delivery system.

We previously reported that RA self-assembled into micelles with ultrasmall particle sizes $(<4 \mathrm{~nm})$ in a homogeneous distribution state (polydispersity index 
[PDI] <0.3). These novel ultrasmall micelles showed an appreciable ability to encapsulate hydrophobic molecules such as Coumarin 6 (a fluorescent marker widely used as a substitute for hydrophobic drugs in in vitro and/or in vivo experiments owing to its high fluorescence activity and biocompatibility). ${ }^{3}$ All our previous findings indicated that RA micelle formulations have great potential to serve as novel nano-drug delivery systems that can improve the bioavailability of hydrophobic drugs. ${ }^{4}$ However, the potential of RA as a nanocarrier of an active pharmaceutical ingredient still requires evaluation.

Investigations of novel nano-drug delivery systems often use curcumin (Cur) as a model drug. Many studies have revealed that Cur has anti-inflammatory and antioxidant properties that can be exploited for a broad range of favorable therapeutic activities. ${ }^{5}$ However, the therapeutic applications of Cur are severely limited by several of its characteristics, most notably its poor chemical/storage stability, low bioavailability due to poor membrane permeation, limited water solubility, rapid metabolism, and pronounced systemic elimination. ${ }^{6}$

Literature shows few research reports on the utilization of stevioside and steviol as nanocarriers, ${ }^{7-12}$ and no report has described the use of RA as a novel drug delivery system. The aim of the present work was to develop an RA-based selfnanomicellizing solid dispersion containing Cur (RA-Cur) that would increase Cur solubility and stability and enhance its antioxidant activity and oral bioavailability for the treatment of ulcerative colitis (UC).

\section{Materials and methods}

\section{Chemical reagents and animals}

Details of the chemical reagents and animal use and the ethics statement for animal experimentation are provided in the Supplementary materials.

\section{Determination of critical micelle concentration (CMC)}

The CMC of RA was determined using a fluorescent probe technique with pyrene as the probe, as previously reported. ${ }^{8}$ The CMC of RA was determined in water, simulated gastric fluid (SGF; $\mathrm{HCl}$ solution, $\mathrm{pH}$ 1.2), and simulated intestinal fluid (SIF; United States Pharmacopeia phosphate buffer solution, $\mathrm{pH}$ 6.8).

\section{Preparation of RA-Cur}

Blank RA self-nanomicellizing solid dispersions of RA-Cur were fabricated from ethanol solutions using a solvent evaporation technique, as previously reported. ${ }^{13}$ Briefly, Cur and RA at different RA/Cur weight ratios were weighed accurately and dissolved in $10 \mathrm{~mL}$ ethanol in a $100 \mathrm{~mL}$ round-bottom flask. For blank RA self-nanomicellizing solid dispersions, the Cur was omitted. The solvent was evaporated at $40^{\circ} \mathrm{C}$ under reduced pressure in a rotary evaporator (Yarong Shanghai, China) revolving at $120 \mathrm{rpm}$ until a thin, dry, powdery film formed on the inner wall of the flask; this procedure usually required about 10 minutes. The film was collected, taking efforts to minimize any loss, and was crushed and screened through a mesh size of 80 to obtain a flowable solid system.

\section{Characterization of RA-Cur}

Morphological characterization

The surface morphology of the RA-Cur in solid state was examined by scanning electron microscopy (SEM) using a JEM-1200EX microscope (JEOL Ltd, Tokyo, Japan), as previously reported. ${ }^{14}$

\section{Physicochemical characterizations of RA-Cur}

RA-Cur was used to test the physical state of RA and Cur in the RA-Cur by infrared (IR), differential scanning calorimetry (DSC), and X-ray diffraction (XRD) analyses. These procedures have been previously described in detail. ${ }^{14}$

\section{Characterization of self-micellizing properties \\ Micelle size and zeta potential}

The size distribution and zeta potential of the RA-Cur micelles in water were determined as described previously. ${ }^{15,16}$ A solution with a Cur concentration of $1.0 \mathrm{mg} / \mathrm{mL}$ was used for this determination.

\section{Morphological characterization}

The morphologies of RA-Cur or the blank formulations in solution were examined by transmission electron microscopy using a JEM-1200EX microscope (JEOL Ltd), as previously reported. ${ }^{14}$

\section{Quantification of Cur and encapsulation efficiency of Cur in RA-Cur}

The Cur content was quantitatively determined with a HPLC system, as previously reported. ${ }^{15}$ RA-Cur was dispersed in water and a transparent micelle dispersion solution was obtained with gentle shaking. The solution was filtered through a $0.22-\mu \mathrm{m}$ filter to remove the insoluble 
(unencapsulated) Cur. The micelle solution was then diluted for analysis of the Cur content by HPLC. The encapsulation efficiency was calculated as the ratio of the amount of Cur detected in the micelles after filtration vs before filtration.

\section{Determination of the apparent solubility of RA-Cur}

The apparent solubility (ie, the solubility measured at 4 hours, not the solubility at equilibrium $)^{17}$ of Cur in the RA-Cur formulation was determined by dispersing RA-Cur samples in water and shaking at $100 \mathrm{rpm}$ on a shaking table for 4 hours at $37^{\circ} \mathrm{C}$. The samples were then centrifuged at $7,378 \times g$ $(10,000 \mathrm{rpm})$ for 10 minutes. The supernatant was filtered through a $0.22-\mu \mathrm{m}$ filter and then analyzed for Cur by HPLC.

\section{Stability of RA-Cur}

The storage stability of RA-Cur was investigated as previously reported, with minor modifications. ${ }^{14,18}$ The RA-Cur solid powder was transferred to glass vials, wrapped in aluminum foil to protect from light, and stored at $4^{\circ} \mathrm{C}$ and $25^{\circ} \mathrm{C}$. One glass vial for each formulation sample was withdrawn at 2-month intervals for evaluation of the encapsulation efficiency by HPLC, as described earlier. The actual level of the encapsulation efficiency in each interval was detected and normalized to its actual encapsulation efficiency at day 0 . The chemical stability of the Cur loaded into the RA-Cur was also assessed by monitoring its chromatographic profile.

\section{Measurement of antioxidant activity}

The antioxidant capacity of Cur was determined by the ferric reducing antioxidant power (FRAP) assay using previously described methods. ${ }^{19,20}$ Free Cur and RA-Cur, at Cur concentrations of 50,100, 200, 250, and $300 \mu \mathrm{g} / \mathrm{mL}$, were tested. The corresponding RA concentrations with the $\mathrm{RA} / \mathrm{Cur}$ weight ratio of $25: 1(1.25,2.50,5.00,6.25$, and $7.50 \mathrm{mg} / \mathrm{mL}$ of RA, respectively) were also tested.

\section{Biodistribution of the preparations in the intestinal tract}

The biodistribution of the RA-Cur formulation was evaluated as described in previous reports. ${ }^{21,22}$ Free Cur and RA-Cur were administered to the rats by an intragastric route at $150 \mathrm{mg} / \mathrm{kg}$ and the rats were sacrificed at 0.5 and 1.5 hours after administration. Different intestinal segments, including duodenum, jejunum, and ileum, were carefully collected. The tissues were further processed as previously reported, ${ }^{21}$ and the Cur contents were analyzed by HPLC. The concentration of Cur in each sample was normalized to the selected tissue weight. The absorption of Cur in tissues was visualized by carefully isolating and excising different intestinal segments, followed by careful washing with PBS, eversion of the selected tissues, and preparation of frozen sections. The cell nuclei were stained blue with 2-(4-amidinophenyl)-6-indolecarbamidine dihydrochloride and F-actin was stained red with rhodamine-phalloidin, following established protocols. The stained tissue sections were observed with an Eclipse TE2000-U fluorescence microscope (Nikon Corporation, Tokyo, Japan).

\section{Pharmacokinetic studies in rats}

Pharmacokinetic evaluations were performed in the rats as reported previously. ${ }^{22,23}$ Twelve rats were randomly divided into two groups and were administered (intragastric administration [ig]) free Cur (free Cur dispersed in an aqueous suspension containing $2.5 \mathrm{mg} / \mathrm{mL}$ carboxymethyl-cellulose and $1 \mathrm{mg} / \mathrm{mL}$ Tween 80) or RA-Cur at a Cur dose of $150 \mathrm{mg} / \mathrm{kg}$. Blood samples $(0.5 \mathrm{~mL})$ were collected and plasma was separated as described previously. ${ }^{23}$ The Cur content of the plasma samples was determined by HPLC.

\section{In vivo anti-colitis activity}

The in vivo anti-colitis activities of RA-Cur preparations were investigated in mice using previously described methods. ${ }^{22,24,25}$ Eight-week-old Kunming male mice were given drinking water containing $35 \mathrm{mg} / \mathrm{mL}$ dextran sodium sulfate (DSS) to establish the UC mouse model. The UC mice were divided into the following six groups, with eight mice per group: DSS control group, RA-treated DSS group (3.75 g RA/kg mouse weight), free Cur-treated DSS group (150 mg Cur/kg mouse weight), RA-Curtreated DSS group (50 mg Cur/kg mouse weight), RA-Curtreated DSS group (100 mg Cur/kg mouse weight), and RA-Cur-treated DSS group (150 mg Cur/kg mouse weight). Eight normal male mice of the same age were used as a healthy control group. Mice in every group were administered (ig) the preassigned agents every day. The time course of the experiments is depicted in Figure S2. At the end of the treatments, the mice were euthanized and their colons and spleens were immediately removed for further measurements. The colon tissues were carefully flushed with PBS. Sections 5 - $\mu \mathrm{m}$-thick were prepared and stained with H\&E for histological evaluation.

\section{Results \\ CMC of RA}

The CMC values for $\mathrm{RA}$ at $37^{\circ} \mathrm{C}$ were $6.23 \pm 1.03$, $5.45 \pm 0.75$, and $5.42 \pm 0.70 \mathrm{mg} / \mathrm{mL}(6.44 \pm 1.07,5.64 \pm 0.78$, and $5.60 \pm 0.72 \mathrm{mM})$ in water, $\mathrm{SGF}(\mathrm{pH} 1.2)$, and $\mathrm{SIF}(\mathrm{pH} 6.8)$, 
respectively, indicating that RA has a strong tendency to form micelles.

\section{Characterization studies of RA-Cur solid dispersion}

RA-Cur was successfully fabricated by the simple method described in this paper. In the solid state, RA-Cur was a uniform yellow powder when viewed with the naked eye (Figure 1A). The morphological characteristics of RA-Cur are shown in Figure 1B. SEM micrographs of unprocessed RA showed agglomerates with slightly bacilliform shape (Figure 1Ba) and unprocessed Cur (Figure 1Bb); the physical mixture of RA and Cur (Figure 1Bc) also showed similar shapes with RA. No obvious changes were observable after RA-Cur fabrication (Figure 1Bd).
The DSC curves obtained are shown in Figure $1 \mathrm{C}$. The RA thermogram displayed a thermal event at $236.67^{\circ} \mathrm{C}$, corresponding to the melting point, while Cur exhibited a melting peak at $183.00^{\circ} \mathrm{C}$. The RA and Cur melting points remained at practically the same temperatures for a physical mixture of RA and Cur. When RA and Cur were formulated into RA-Cur, the peak corresponding to the Cur melting point disappeared, and the RA melting range was extended, indicating that Cur was present within the solid dispersion in an amorphous state.

The IR spectrum obtained is shown in Figure 1D. The Cur IR spectrum showed cis- $\mathrm{CH}$ vibrations of the aromatic ring evident at $817 \mathrm{~cm}^{-1}$. Benzoate trans-CH vibrations were visible at $964 \mathrm{~cm}^{-1}$. The stretching of the carbonyl group and $=\mathrm{C}-\mathrm{O}-\mathrm{C}$ was visible as the peaks at 1,514 and
A
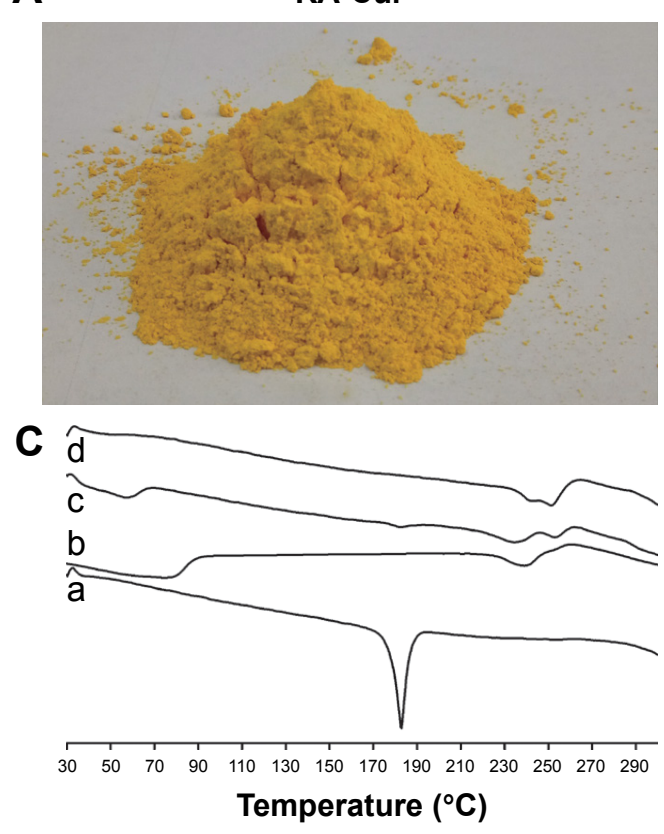

D

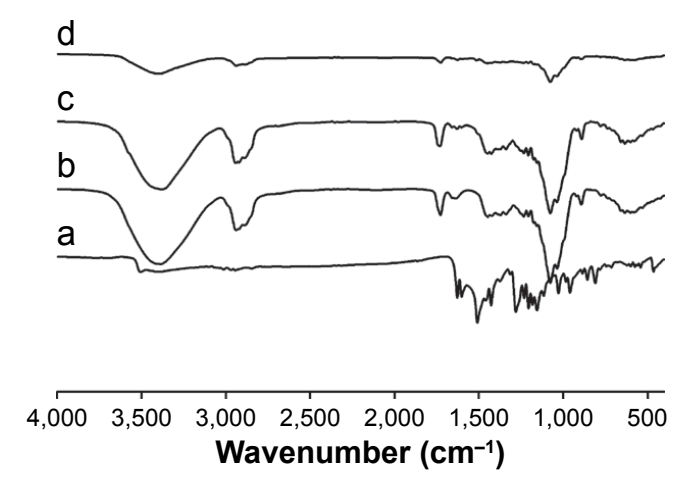

B
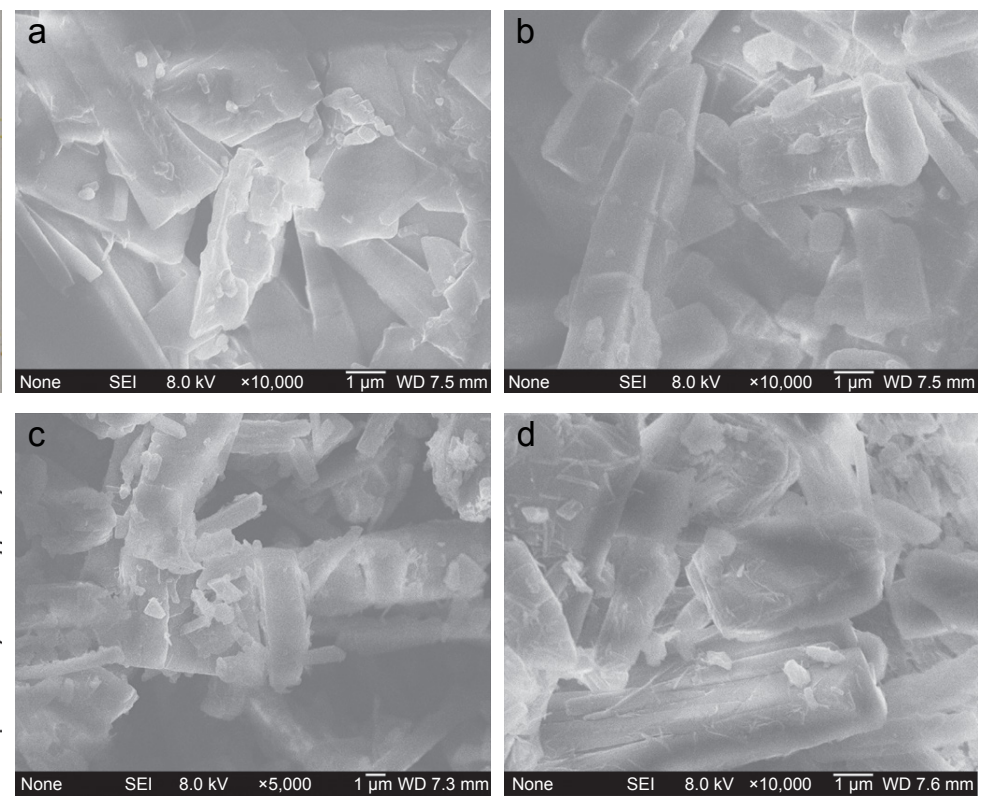

E

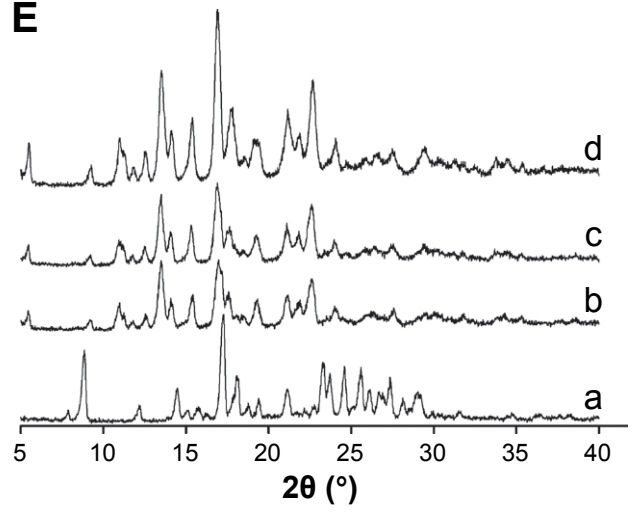

Figure I Physicochemical properties of RA-Cur with RA/Cur weight ratio of 25:I in solid state.

Notes: (A) RA-Cur appearance; (B) SEM micrographs; (C) thermal analysis; (D) IR spectra; (E) XRD analysis. (a) Cur, (b) RA, (c) physical mixture of RA and Cur, and (d) RA-Cur. Abbreviations: Cur, curcumin; IR, infrared; RA, rebaudioside A; RA-Cur, RA-based self-nanomicellizing solid dispersion containing Cur; SEM, scanning electron microscopy; $\mathrm{XRD}, \mathrm{X}$-ray diffraction. 
$1,086 \mathrm{~cm}^{-1}$, respectively. Vibrations of $\mathrm{C}=\mathrm{C}$ and $\mathrm{C}=\mathrm{O}$ bonds combined in a strong peak at $1,633 \mathrm{~cm}^{-1}$. A sharp peak at $3,523 \mathrm{~cm}^{-1}$ indicated the presence of $-\mathrm{OH}$ groups.

The spectrum for RA showed the following: $-\mathrm{C}=\mathrm{O}$ vibrations and a $\mathrm{C}-\mathrm{O}-\mathrm{C}$ fragment were evident at 1,727 and $1,076 \mathrm{~cm}^{-1}$, respectively. The $-\mathrm{OH}$ vibrations were visible at $3,387 \mathrm{~cm}^{-1}$ and the $-\mathrm{CH}_{3} /-\mathrm{CH}_{2} /-\mathrm{CH}$ fragment was visible at $2,939 \mathrm{~cm}^{-1}$. Comparison of the spectrum of RA-Cur with that of the physical mixtures of RA and Cur revealed no new absorption peaks, indicating that no chemical reactions had occurred during the sample preparation procedures.

The XRD spectrum obtained is shown in Figure 1E. XRD analysis of Cur revealed several high-intensity and sharp peaks at $8.84^{\circ}, 14.48^{\circ}, 17.26^{\circ}, 18.06^{\circ}, 21.14^{\circ}, 23.28^{\circ}, 24.60^{\circ}$, $25.6^{\circ}$, and $27.34^{\circ}$, indicating that Cur was in a crystalline state. The diffraction patterns of RA also corresponded to a crystalline state, with sharp, high-intensity peaks appearing at $13.48^{\circ}, 14.08^{\circ}, 15.42^{\circ}, 16.92^{\circ}, 19.34^{\circ}, 21.18^{\circ}, 21.90^{\circ}$, and $22.64^{\circ}$. The patterns for the physical mixture of RA and Cur revealed all the characteristic peaks of both RA and Cur. The RA-Cur diffraction pattern was similar to that of the crystalline structure of RA. The spectra did not show any of the typical Cur peaks, indicating a noncrystalline state of Cur in RA-Cur.

The RA/Cur weight ratio was an important factor in the preparation of RA-Cur. RA exhibited a low CMC and an ultrasmall micelle size in a homogeneous distribution state in aqueous solution, and the RA micelles showed a high efficiency for the encapsulation of Cur. This encapsulation was as high as $99.43 \% \pm 0.67 \%$ for Cur at an RA/Cur weight ratio of 10:1, indicating almost complete encapsulation. However, the stability showed obvious variations when different RA/Cur weight ratios were tested (Figure 2). Storage at both $4^{\circ} \mathrm{C}$ and $25^{\circ} \mathrm{C}$ with protection from light revealed a very slow and time-dependent leakage of Cur from RA-Cur. Overall, $98.24 \% \pm 0.37 \%$ of the Cur in the $25: 1$ RA/Cur weight ratio preparation and $95.84 \% \pm 1.79 \%$ of the Cur in the 20:1 RA/Cur weight ratio preparation remained encapsulated in the RA micelle formulations after 6 months of storage at $4^{\circ} \mathrm{C}$. By contrast, formulations with RA/Cur weight ratios of 15:1 and 10:1 readily dissolved in water after 6 months of storage at $4^{\circ} \mathrm{C}$, but the Cur precipitated within several minutes, indicating that these preparations were unstable during storage. The Cur leakage from the RA-Cur formulations with different RA/Cur weight ratios was more pronounced following storage at $25^{\circ} \mathrm{C}$ than at $4^{\circ} \mathrm{C}$. After 6 months of storage at $25^{\circ} \mathrm{C}, 93.93 \% \pm 1.36 \%$ of the Cur remained encapsulated in the RA micelle formulation with a 25:1 RA/Cur weight ratio, while $91.73 \% \pm 6.55 \%$ of the Cur remained encapsulated for the formulation with the 20:1 RA/Cur weight ratio. The formulations with RA/ Cur weight ratios of 15:1 and 10:1 were equally unstable after 6 months of storage at either $25^{\circ} \mathrm{C}$ or $4^{\circ} \mathrm{C}$.

These results indicated that the RA-Cur formulations with high RA/Cur weight ratios were stable when stored at $4^{\circ} \mathrm{C}$. The formulation with a 25:1 RA/Cur weight ratio was therefore chosen for further tests of encapsulation efficiency and storage stability. The micelle size, PDI, and zeta potential of RA-Cur with a 25:1 weight ratio were $4.186 \pm 0.231 \mathrm{~nm}$, $0.194 \pm 0.013$, and $-1.873 \pm 0.494 \mathrm{mV}$, respectively (Figure 3).

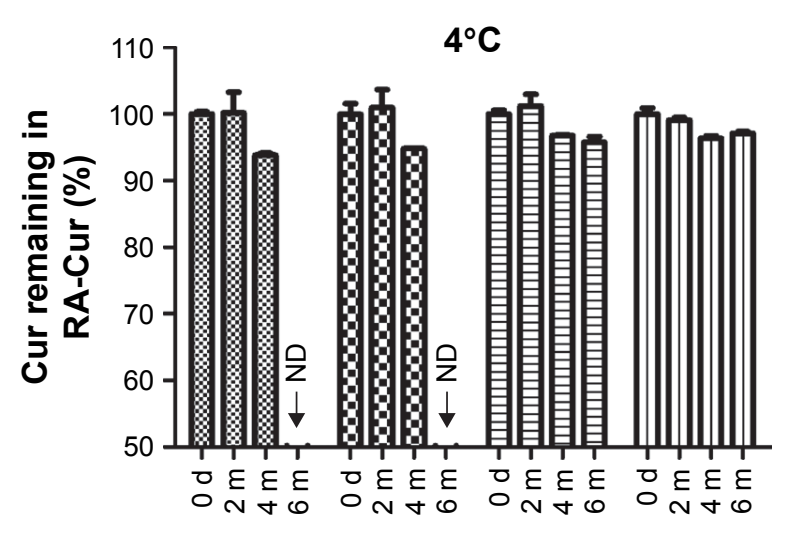

Time

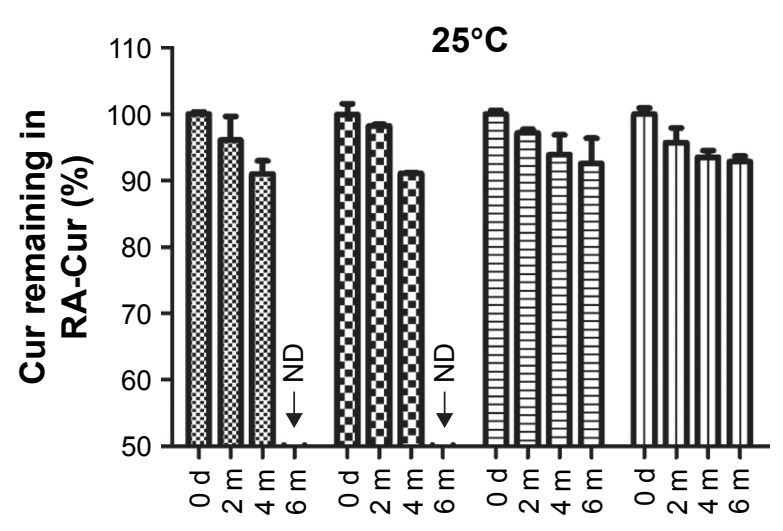

Time

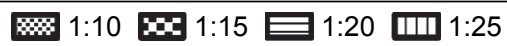

Figure 2 RA-Cur stability.

Notes: RA-Cur stored at $4^{\circ} \mathrm{C}$ and $25^{\circ} \mathrm{C}$ with protection from light. At 2-month intervals, RA-Cur was dissolved in water and its encapsulation efficiency was determined by HPLC. Cur remaining in RA-Cur (\%) was expressed as the actual level of the encapsulation efficiency to each interval normalized to its actual encapsulation efficiency at day 0 ( $n=3$ ). Abbreviations: Cur, curcumin; ND, not determined; RA, rebaudioside A; RA-Cur, RA-based self-nanomicellizing solid dispersion containing Cur. 
A

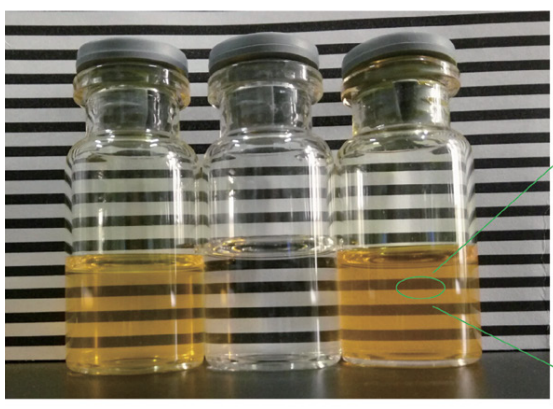

Cur in ethanol Water RA-Cur in water

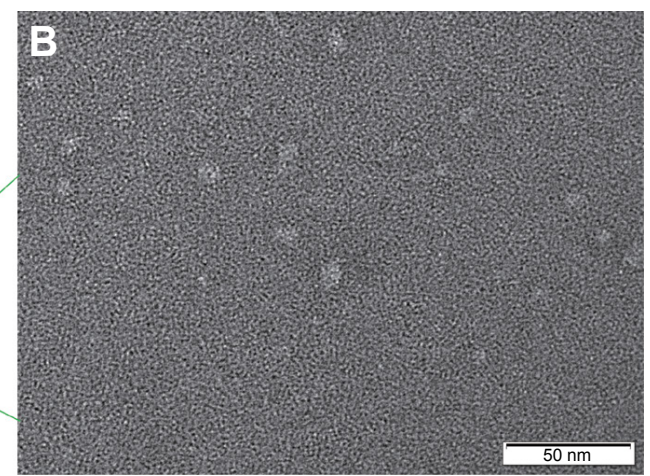

231.21

C

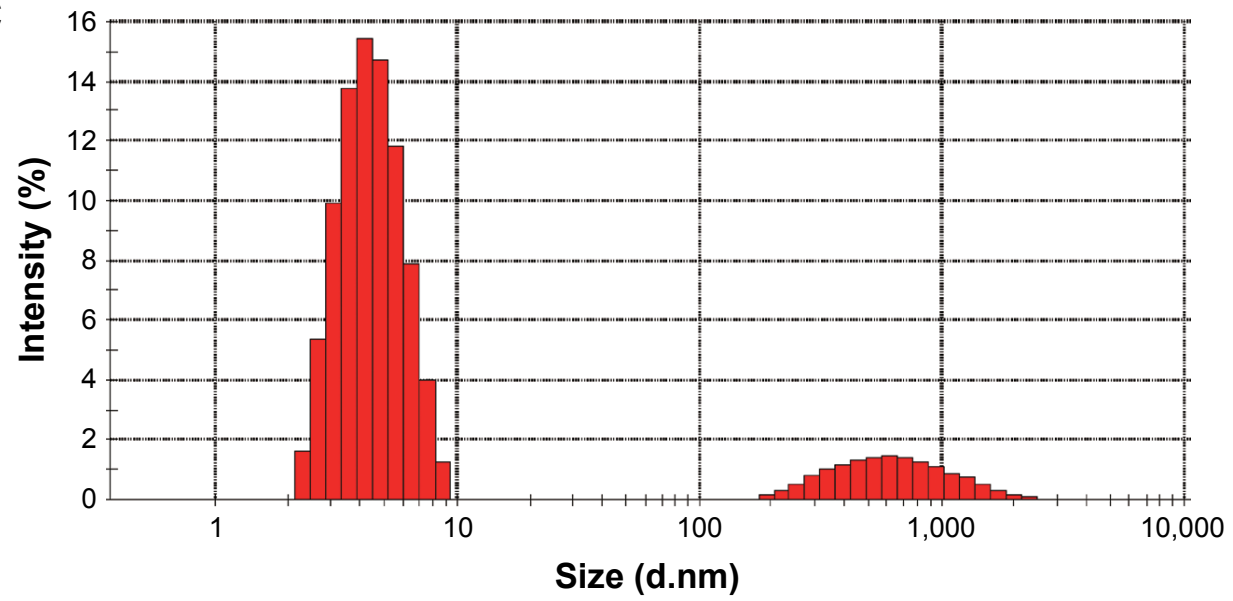

D

$\begin{array}{cclll} & & \text { Mean }(\mathrm{mV}) & \text { Area }(\%) & \text { Width }(\mathrm{mV}) \\ \text { Zeta potential }(\mathrm{mV}):-5.82 & \text { Peak 1: } & 0.00 & 0.0 & 0.00 \\ \text { Zeta deviation }(\mathrm{mV}): 0.00 & \text { Peak 2: } & 0.00 & 0.0 & 0.00 \\ \text { Conductivity }(\mathrm{mS} / \mathrm{cm}): 20.4 & \text { Peak 3: } & 0.00 & 0.0 & 0.00\end{array}$

Figure 3 Characterization of RA-Cur with an RA/Cur weight ratio of 25:I in water.

Notes: (A) The appearance of the RA-Cur micelle solution; (B) TEM morphology of RA-Cur micelles $(\times 400 \mathrm{k}$ magnification, bar =50 nm); (C) micelle size distribution; (D) zeta potential characterization of the RA-Cur micelle solution (RA/Cur weight ratio of 25:I).

Abbreviations: Cur, curcumin; RA, rebaudioside A; RA-Cur, RA-based self-nanomicellizing solid dispersion containing Cur; TEM, transmission electron microscopy.

A bimodal size distribution was observed for RA-Cur after it was dissolved in water. The small-size distribution could be explained as the RA micelles encapsulating Cur and large size ascribed to the unencapsulated Cur. The apparent solubility was $14.34 \pm 1.66 \mathrm{mg} / \mathrm{mL}$ for this formulation.

\section{Antioxidant activity of RA-Cur}

As shown in Figure 4A, the antioxidant activities (reduction of $\mathrm{Fe}^{3+}$-tripyridyltriazine to $\mathrm{Fe}^{2+}$-tripyridyltriazine) of free Cur and RA-Cur determined at different time points were enhanced at concentrations ranging from 50 to $300 \mu \mathrm{g} / \mathrm{mL}$, indicating concentration-dependent antioxidant activities of free Cur and RA-Cur. As shown in Figure 4B, for different Cur concentrations, the antioxidant activities of both free Cur and RA-Cur increased when the reaction time was increased from 15 to 120 minutes, indicating time-dependent antioxidant activities of free Cur and RA-Cur. However, the antioxidant activity was stronger for RA-Cur than for free Cur. RA alone showed no antioxidant activity in this test.

\section{Biodistribution of Cur in the intestinal tract}

The biodistribution profiles of free Cur and RA-Cur in the intestinal tract were studied with both quantitative and fluorescence methods. The absorption profiles of Cur in the free Cur and RA-Cur groups differed in terms of the site in the intestinal segments, as shown in Figure 5A and D. For the RA-Cur group at the 0.5 -hour time point, the rank order 

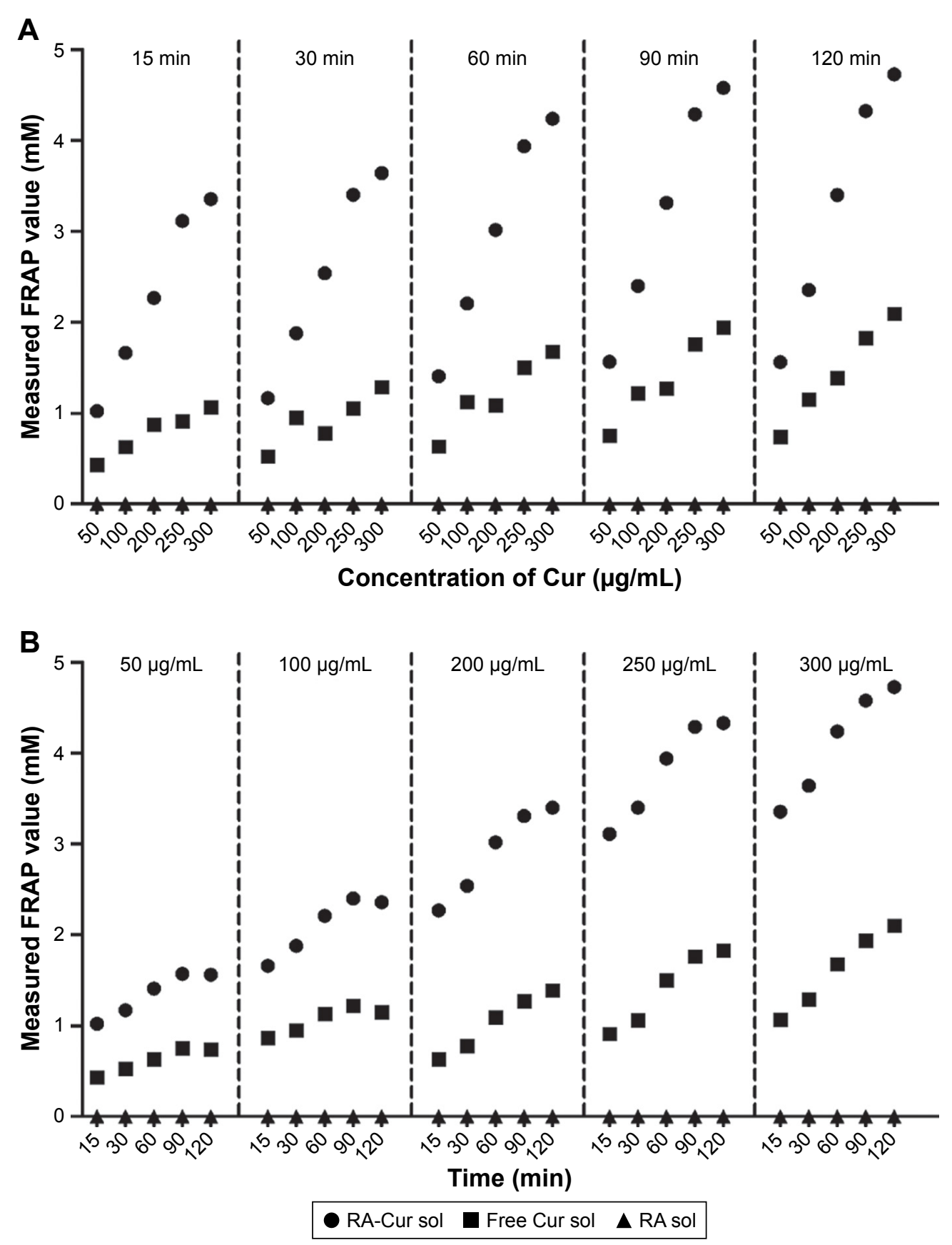

Figure 4 Measured FRAP values.

Note: Measured FRAP values of the RA solution, free Cur, and RA-Cur (A) at different concentrations as a function of time, and (B) with different incubation times as a function of concentration $(n=3)$.

Abbreviations: Cur, curcumin; FRAP, ferric reducing antioxidant power; RA, rebaudioside A; RA-Cur, RA-based self-nanomicellizing solid dispersion containing Cur; sol, solvent.

of the concentrations of Cur in different intestinal segments was duodenum $>$ jejunum $>$ ileum, and this difference was attributed to the different physiological features of the various selected segments. However, the concentrations of Cur in these three different intestinal segments in the free Cur group were found to be similar $(P>0.05)$. When compared to the free Cur group, the RA-Cur group showed significantly enhanced intestinal absorption of Cur. The Cur concentration in the segments of duodenum and jejunum in the free Cur group was decreased while the concentration in the ileum increased after 1.5 hours. By contrast, dramatic increases were observed in all three segments of the RA-Cur group at the 1.5-hour time point. The Cur concentrations in the duodenum, jejunum, and ileum were 38.01-, 11.55-, and 

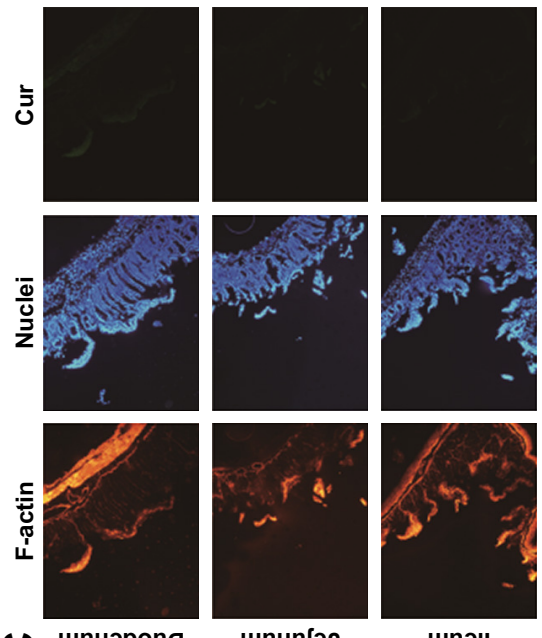

unun!๋̣

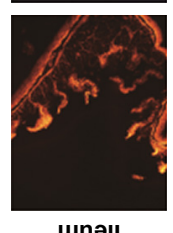

$\circlearrowleft$ unuəpong
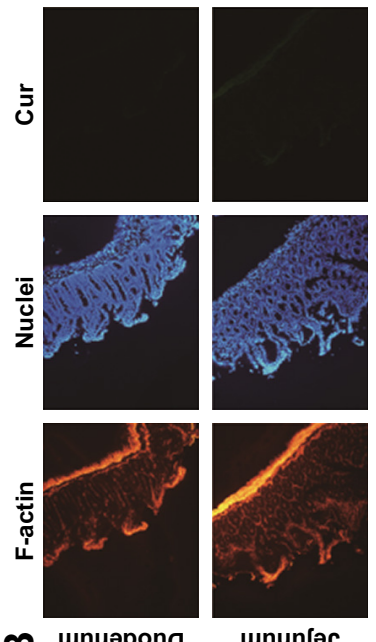

ununโ̣「
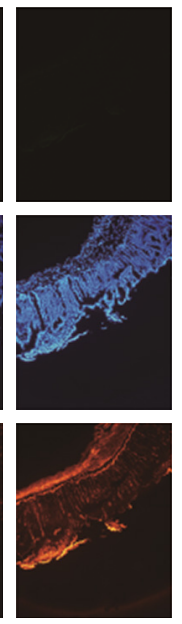

unฑા

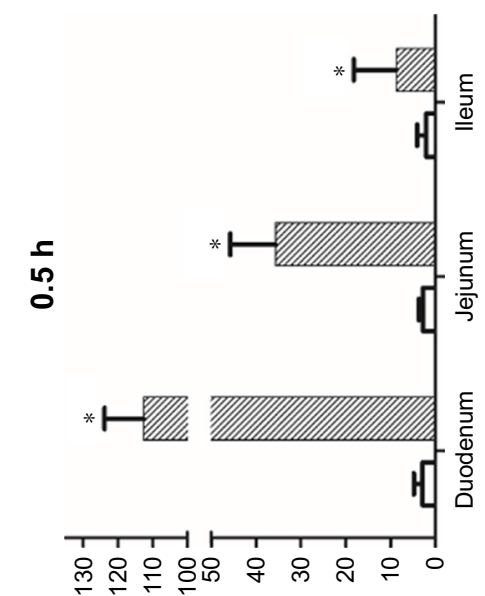

(6u/6u) әnss!ł u!

$\varangle \quad$ Ino jo uo!łedłuəouos

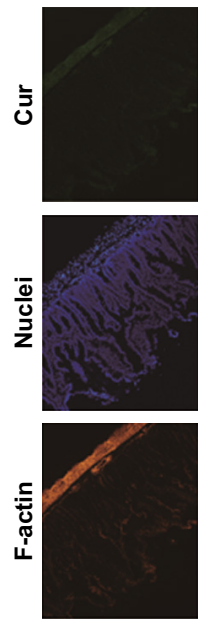

L. unuəpona
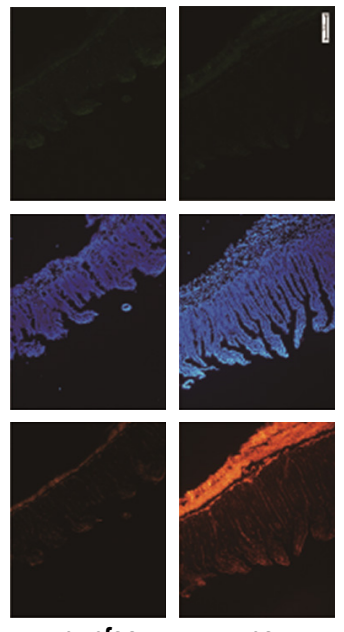

unə|
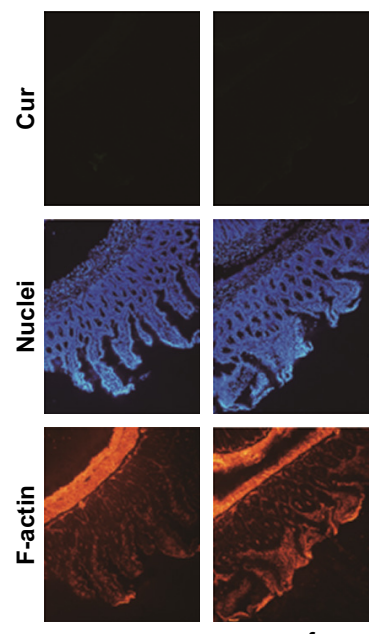

II unuəpong
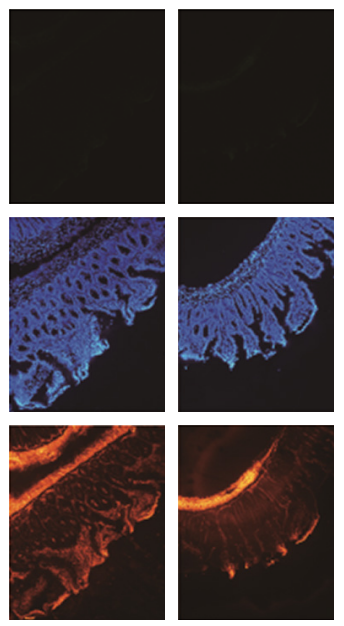

ununโ̣|ər

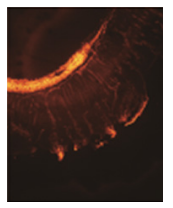

unข|

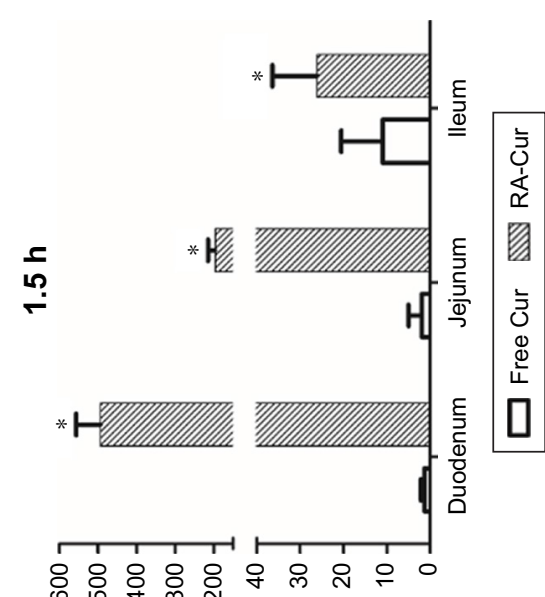

(6u/bu) әnss!! u!

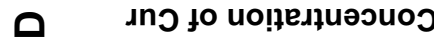

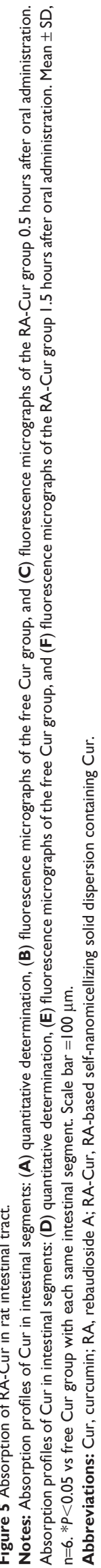


2.99-fold higher, respectively, in the RA-Cur group than in the Cur group at 0.5 hours, and 352.11-, 96.84-, and 1.36-fold higher, respectively, at 1.5 hours.

The fluorescence observations of different intestinal segments at the 0.5 - and 1.5-hour time points after oral administration of free Cur and RA-Cur are shown in Figure 5C and F, respectively. The rank order of the green fluorescence signal (corresponding to RA-Cur or free Cur molecules released from the RA-Cur) in the different intestinal segments (duodenum $>$ jejunum $>$ ileum) reflected the quantitative determination results. Stronger Cur fluorescence was observed in intestinal epithelial cells of the RA-Cur group and was accompanied by deeper penetration when compared with the Cur group at either 0.5 or 1.5 hours. These observations could indicate Cur uptake primarily from the intestinal lumen and prompt absorption into the duodenum, followed by absorption into the jejunum and ileum. Green fluorescence was also evident in the mucous membrane and in the serosa membrane, deep inside the intestine. These results indicated that RA-Cur (or the Cur released from RA-Cur) was able to penetrate through the mucus layer, reach the serosa membrane, and subsequently be absorbed into the systemic circulation system. The fluorescence intensity was much lower in the free Cur group (Figure 5B and E). These fluorescence observations were consistent with the quantitative Cur uptake results.

\section{Pharmacokinetic studies}

The pharmacokinetic profiles were evaluated in rats to determine the oral absorptive efficiency of RA-Cur. The mean plasma Cur concentration-time profiles are shown in Figure 6. The plasma concentration of Cur was dramatically increased in the RA-Cur group when compared with the

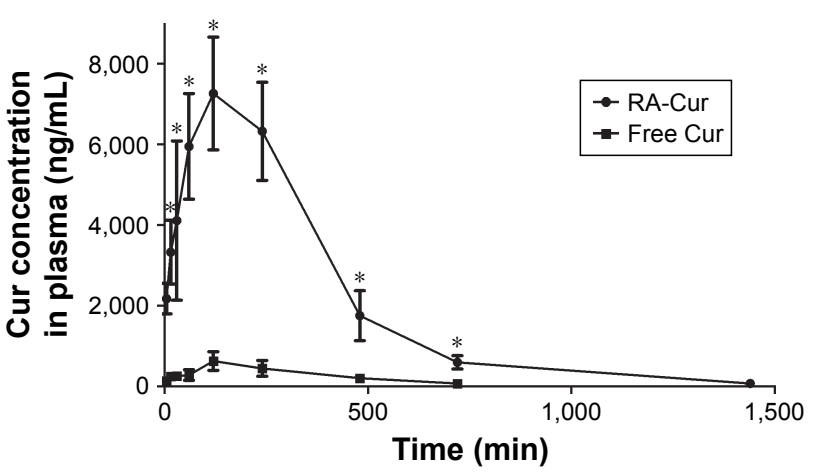

Figure 6 Pharmacokinetic studies.

Note: Pharmacokinetics profiles of RA-Cur and free Cur $(* P<0.05$ vs free Cur group at each same time point) (mean $\pm S D, n=6$ ).

Abbreviations: Cur, curcumin; RA-Cur, RA, rebaudioside A; RA-based selfnanomicellizing solid dispersion containing Cur. free Cur group. Figure 6 also shows that the plasma Cur concentration was higher in the RA-Cur group than in the free Cur group at all time points throughout the experiment. In fact, the plasma Cur concentration in the free Cur group was below the detection limits after 12 hours. The pharmacokinetic parameters (Table S1) indicated an 11.59-fold higher $\mathrm{C}_{\max }$ value for the RA-Cur group than for the free Cur group $(P<0.05)$. The time of maximum concentration $\left(\mathrm{T}_{\max }\right)$ was unchanged, but the $T_{1 / 2}$ value decreased from 3.62 hours for the Cur suspension to 2.78 hours for RA-Cur. The significantly increased $\mathrm{C}_{\max }$ value and decreased $T_{1 / 2}$ value could be explained by the improvement in solubility and faster release of Cur from the RA-Cur due to the RA micelle encapsulation. The area under the curve $\left(\mathrm{AUC}_{0-t}\right)$ value was 19.06-fold greater for the RA-Cur group when compared to the free Cur group.

\section{In vivo therapeutic activity Body weight loss}

As shown in Figure 7A, the body weight in the healthy control group showed a normal increase $(+9.32 \%)$ as the experimental period progressed. By contrast, the body weight of mice in the DSS control group showed a significant decrease at the end of the test $(-15.87 \%$, compared to the healthy control group, $P<0.05)$, and the RA treatment failed to prevent this decrease in body weight $(-13.52 \%$, compared to the DSS control group, $P>0.05$, and $P<0.05$ to the healthy control group). On the contrary, administration of free Cur at $150 \mathrm{mg} / \mathrm{kg}$ almost completely abolished this decrease $(-0.18 \%$, compared to the DSS control group or healthy control group, $P<0.05)$. Administration of RA-Cur at 50 and $100 \mathrm{mg} / \mathrm{kg}$ RA-Cur completely abolished the body weight decrease $(+0.78 \%$ and $+0.97 \%)$, giving efficacies similar to that achieved with $150 \mathrm{mg} / \mathrm{kg}$ free $\mathrm{Cur}(P>0.05)$. The body weight increased by $4.50 \%$ in the treatment group given $150 \mathrm{mg} / \mathrm{kg}$ RA-Cur (compared to the DSS control group or the healthy control group, $P<0.05$ ).

\section{Colon length}

The colon lengths (Figure 7B) exhibited a similar pattern to body weight and spleen weight in the different mouse groups. Colon length shortening was much more severe in the DSS group than in the healthy control group and the treatment groups. RA treatment resulted in some restoration of the colon length, but it was less effective than the free Cur or RA-Cur treatment. The $150 \mathrm{mg} / \mathrm{kg}$ Cur treatment effectively restored the colon length to $9.53 \mathrm{~cm}$, which was close to that of the healthy control group $(10.46 \mathrm{~cm}$, compared to healthy 

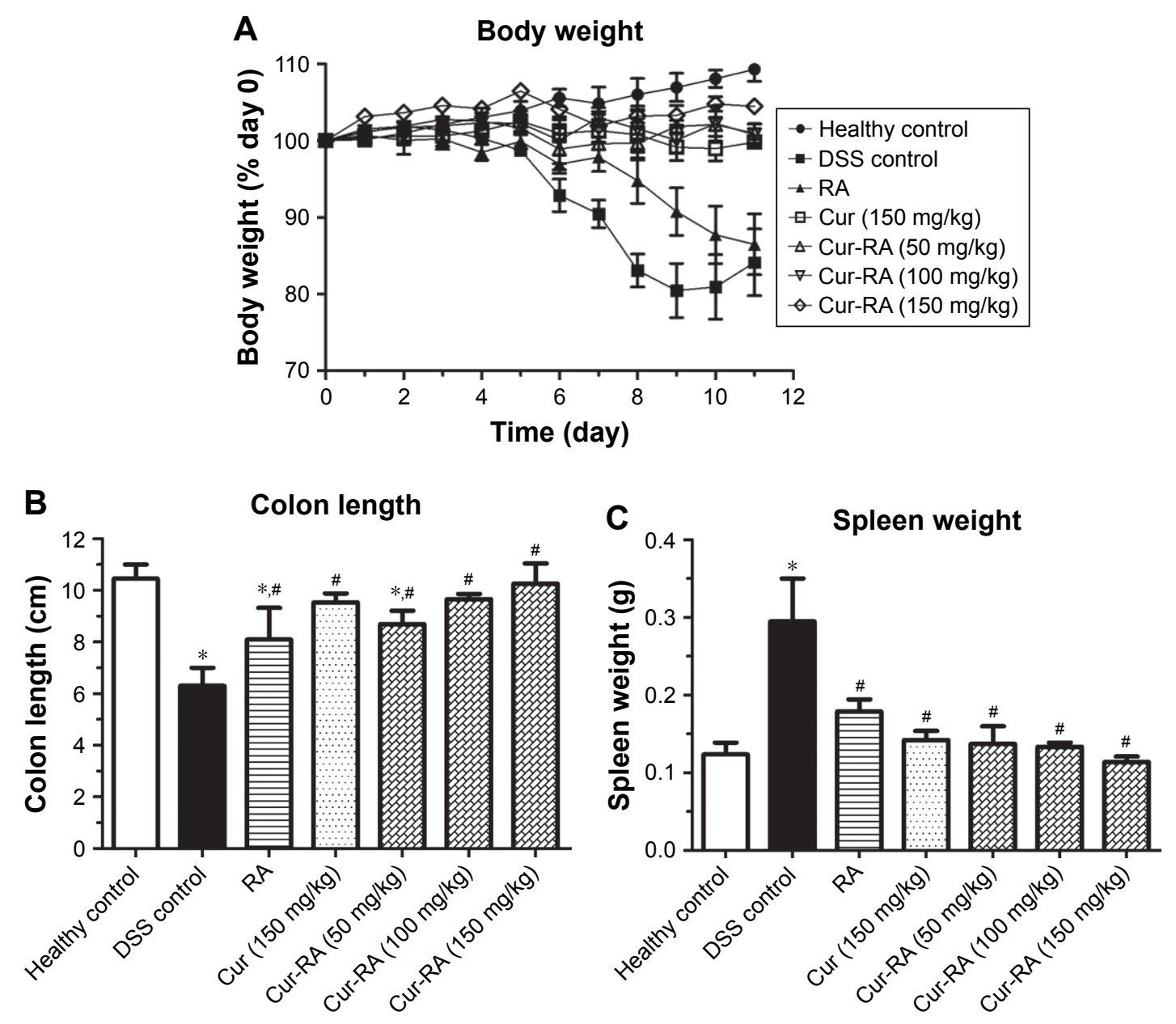

Figure 7 Therapeutic effects of oral administration of RA-Cur on UC in mice induced by DSS.

Notes: (A) Body weight change profiles. Mouse body weight was measured throughout the experimental duration, and it was normalized as a percentage of the day 0 body weight. The colon and spleen were harvested on day I2. (B) Colon length and (C) spleen weight in different mice groups were measured. $* P<0.05$ vs healthy control group, ${ }^{\#} P<0.05$ vs DSS control group. Each point represents the mean \pm SD $(n=8)$.

Abbreviations: Cur, curcumin; DSS, dextran sodium sulfate; RA, rebaudioside A; RA-Cur, RA-based self-nanomicellizing solid dispersion containing Cur; UC, ulcerative colitis.

control group, $P>0.05)$. The RA-Cur treatment showed concentration-dependent effects, and the $150 \mathrm{mg} / \mathrm{kg}$ treatment completely restored the colon length to a length $(10.27 \mathrm{~cm})$ similar to that of the healthy control group $(P>0.05)$.

\section{Spleen weight}

The spleen weight profiles are shown in Figure 7C. The healthy control group had a mean spleen weight of $0.12 \mathrm{~g}$, whereas, interestingly, the DSS control group had a significantly higher mean spleen weight $(0.29 \mathrm{~g})(P<0.05$ when compared to the healthy control group). The RA-treated DSS group had a much lower mean spleen weight of $0.18 \mathrm{~g}$ when compared with the DSS control group $(P<0.05)$, although it was still much higher than that of the healthy control group $(P<0.05)$. The $150 \mathrm{mg} / \mathrm{kg}$ Cur treatment also effectively decreased the mean spleen weight to $0.14 \mathrm{~g}$ (compared to the healthy control group, $P>0.05$ ). All three dosages of RA-Cur resulted in spleen weights similar to that of the healthy control group (mean spleen weights of $0.14,0.13$, and $0.11 \mathrm{~g}$ for mice treated with 50,100 , and $150 \mathrm{mg} / \mathrm{kg}$ RA-Cur, respectively; $P>0.05$ when compared to the healthy control group).

\section{Histopathologic examination}

Histopathologic examinations of colon tissues were performed to further confirm the therapeutic efficacy of RA-Cur against DSS-induced UC (Figure 8). The healthy control group showed normal colon histology, including normal glands, dense microvilli, and abundant goblet cells, and no sign of inflammation or disruption of colon tissue morphology. However, colon tissues from the DSS control group exhibited obvious microvillus atrophy and a small number of glands 

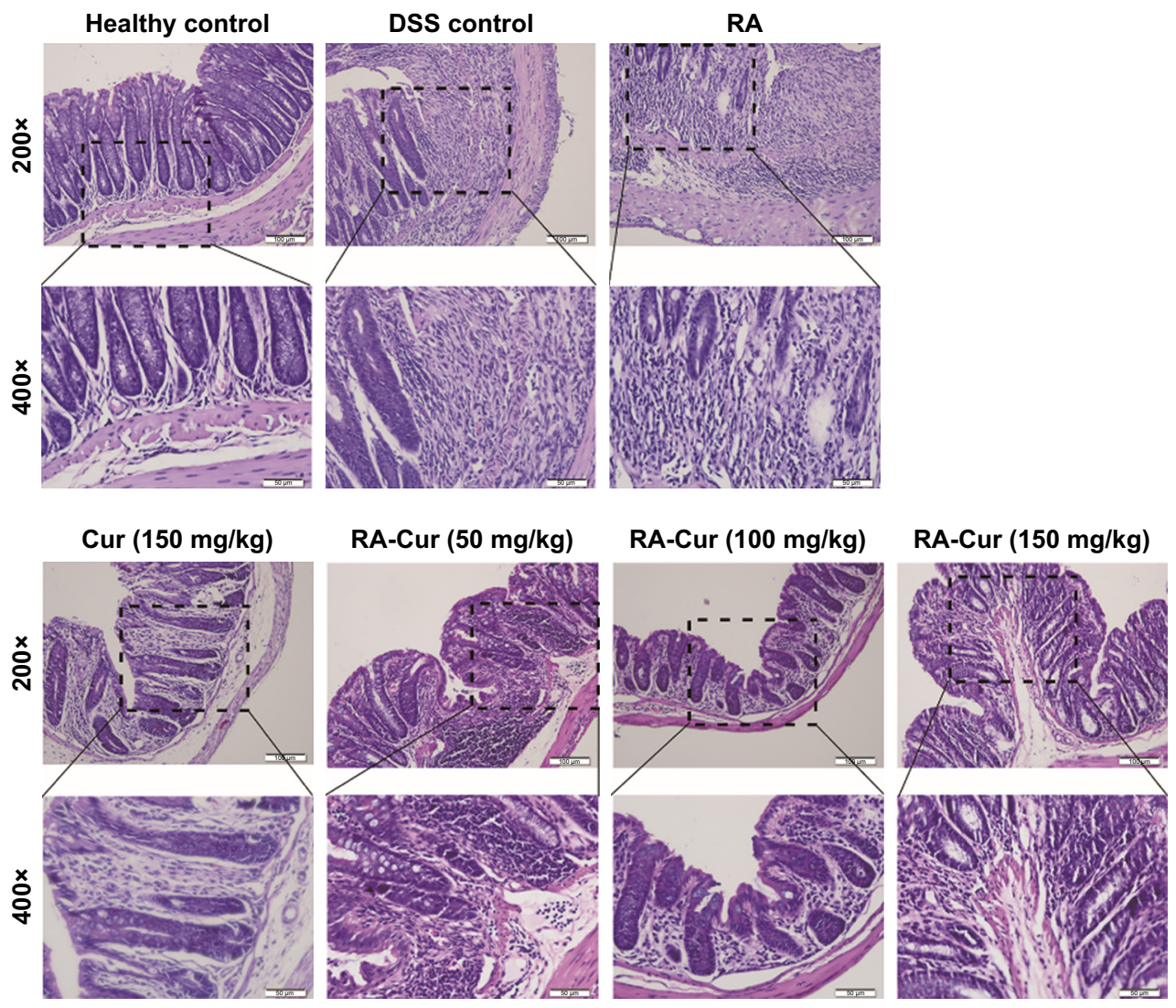

Figure 8 Histopathologic examinations of colon tissues.

Notes: Representative colon sections from the healthy control group, DSS control group, RA-treated group, Cur I50 mg/kg-treated group, RA-Cur 50 mg/kg-treated group, RA-Cur $100 \mathrm{mg} / \mathrm{kg}$-treated group, and RA-Cur $150 \mathrm{mg} / \mathrm{kg}$-treated group. Scale bar to $200 \times=100 \mu \mathrm{m}$. Scale bar to $400 \times=50 \mu \mathrm{m}$.

Abbreviations: Cur, curcumin; DSS, dextran sodium sulfate; RA, rebaudioside A; RA-Cur, RA-based self-nanomicellizing solid dispersion containing Cur.

distributed in the numerous fibroblastic proliferations. These colon tissues also showed obvious symptoms of inflammation, such as massive infiltration of inflammatory cells (eg, leukocytes and neutrophils). Other observations included significant disruption of the colon epithelium, depletion of goblet cells, crypt distortion, and mucosal ulceration and erosion. Treatment with RA did not provide any marked protection against this colon damage. The Cur $150 \mathrm{mg} / \mathrm{kg}$ treatment group showed a slight improvement in colon histology, but microvillus atrophy and significant depletion of goblet cells were still evident. Glands were distributed in the fibroblastic proliferations, and massive infiltration of inflammatory cells was observed.

The three RA-Cur groups showed dose-dependent improvements in colon histology. The RA-Cur $50 \mathrm{mg} / \mathrm{kg}$ group showed the same symptoms as the DSS control group, including obvious microvillus atrophy, significant depletion of goblet cells, glands distributed in the fibroblastic proliferations, and massive infiltration of inflammatory cells. By contrast, the RA-Cur $100 \mathrm{mg} / \mathrm{kg}$ group showed less severe microvillus atrophy, depletion of goblet cell numbers, an irregular arrangement of the colon glands, and reduced infiltration of inflammatory cells. The RA-Cur $150 \mathrm{mg} / \mathrm{kg}$ group showed a significant improvement in colon histology, as the tissue morphology was much closer to that of the healthy colon tissue, with only slight evidence of microvillus atrophy, depletion of goblet cells, and infiltration of inflammatory cells.

\section{Discussion}

Self-nanomicellizing solid dispersion is a novel formulation that combines the advantages of a solid dispersion-based strategy and nanotechnology as it forms nanosized micelles upon contact with an aqueous medium. ${ }^{26-28}$ This strategy was successfully utilized to fabricate RA-Cur micelles in the present study. To the best of our knowledge, this work is the first to investigate and confirm the potential role of RA as an oral nano-drug delivery system. When used for the oral administration of Cur, a hydrophobic drug, RA enhanced drug solubility and improved delivery by self-nanomicellizing and 
acting as a shield that protected the encapsulated active agents from direct interaction with the aggressive gastrointestinal tract fluids. The results of this study show that RA is a promising excipient for the formulation of self-nanomicellizing solid dispersions with high encapsulation efficiency, as it dissolves quickly and self-assembles into nanomicelles. RA shows great potential for fabrication of formulations with Cur, a hydrophobic drug widely used as a model drug, where improvements in solubility, stability, and bioavailability are urgently needed. ${ }^{29}$

Development and optimization experiments confirmed that RA-based self-nanomicellizing formulations had a strong capacity to encapsulate Cur. However, the RA/Cur weight ratio was not one of the most critical influences on the encapsulation efficiency, as even the RA/Cur weight ratio of $5 / 1$ achieved an encapsulation efficiency as high as $95.70 \%$ (unpublished data). The RA/Cur weight ratio also did not critically influence the micelle size, PDI, or zeta potential, as these three parameters showed no significant variances among formulations with different RA/Cur weight ratios. Notably, these three parameters also showed no significant variations among the four RA/Cur weight ratios tested here (unpublished data). The RA/Cur weight ratio had the strongest influence on storage stability, as the formulation with an RA/Cur weight ratio of 25/1 was more stable than that with a ratio of $20 / 1$ when stored at either $4^{\circ} \mathrm{C}$ or $25^{\circ} \mathrm{C}$, while formulations with ratios of $10 / 1$ and $15 / 1$ were unstable after 6 months. This might reflect the fact that RA-Cur was a solid dispersion, with the amorphous Cur in a solid solution state completely dissolved in the solvent (which was RA in this case). This would result in a high-energy state and an enhanced dissolution rate. However, this is a metastable state from a thermodynamic point of view, and devitrification would eventually take place. ${ }^{30}$ The formulations with low RA/Cur weight ratios (15/1 and 10/1) had less RA available to encapsulate and dissolve the Cur, so the devitrification would be much faster, as indicated by the decreased solubility and immediate Cur precipitation after mixing with water.

Cur loading into the RA-Cur was confirmed by its chemical stability, as a comparison of the chromatographic profiles of RA-Cur at different time points to those of day 0 revealed no new chromatographic peaks. The DSC, XRD, and IR properties of RA-Cur indicated amorphous Cur in RA-Cur. One possibility is that the information about Cur might be obscured by RA in the formulation with the $25: 1$ ratio of RA to Cur. However, IR analysis of a formulation of RA/Cur with a weight ratio of 5/1, and therefore with a higher percentage of Cur, also confirmed the presence of amorphous Cur in the RA-Cur (Figure S3).
The antioxidant activity of encapsulated Cur in RA-Cur was quantified with the FRAP assay and compared to the antioxidant activity of a freshly prepared mixture of free Cur and free RA in PBS. RA-Cur displayed significantly greater antioxidant activity than the mixture, and the drastic improvement in water solubility of Cur in RA-Cur may be one of the important factors. The apparent water solubility of Cur was increased to $14.34 \pm 1.66 \mathrm{mg} / \mathrm{mL}$, when compared with the estimated water solubility of $11 \mathrm{ng} / \mathrm{mL} .{ }^{31}$ Therefore, the formation of RA-Cur micelles could increase the Cur solubility by at least $1.3 \times 10^{6}$-fold, as previously reported. ${ }^{29}$ Contrary to previous reports, ${ }^{1,32}$ we found no obvious antioxidant activity for RA in the present study, indicating the need for further study with more sensitive methods to evaluate the actual antioxidant activity of RA.

RA-Cur had an ultrasmall particle size $(\sim 4 \mathrm{~nm})$ and showed a narrow size distribution (PDI $<0.3$ ). We already confirmed that ultrasmall micelles with narrow size distributions from RA favor deeper penetration into eye tissues. ${ }^{4}$ It has also been widely accepted that particle size has a profound effect on the gastrointestinal tract absorption and biodistribution. ${ }^{33}$ Studies have shown higher uptake of particles with mean diameters of 50-100 $\mathrm{nm}$ in the rat intestine as compared with larger particles. ${ }^{34,35}$ The oral absorption characterizations of ultrasmall particle diameters $<20 \mathrm{~nm}$ have also been revealed in a few reports, and confirmed great improvements in oral bioavailability. ${ }^{36,37}$ Thus, the nano-topographic features of RA-Cur used in this study may significantly encourage gastrointestinal tract absorption and oral bioavailability.

The in vitro rate and extent of Cur release were both significantly improved by encapsulation into RA micelles (Figure S4) and again may reflect the substantial improvement in water solubility of Cur in RA-Cur. The ultrasmall size of the RA micelles also means a sharply increased surface area exposed to the release solution. For these reasons, the in vitro drug release was markedly enhanced in the RA-Cur formulation.

Membrane permeation was 28.9-fold faster for RA-Cur than for free Cur (Figure S5), as determined by Parallel Artificial Membrane Permeability Assay, a model specifically designed to measure passive membrane permeability. This permeation is energy independent, ${ }^{38}$ and it provides permeability values that are useful for oral absorption predictions, because the majority of drugs are absorbed by passive diffusion through the membrane. ${ }^{39-41}$ The improvement in solubility and dissolution (in vitro release) of Cur in RA-Cur contributed to the enhanced permeation. These results suggested that the oral bioavailability of Cur would be improved in in vivo experiments using RA-Cur. 
Cur detection and the fluorescence observations confirmed the RA-Cur biodistribution in the intestinal tract; however, RA-Cur and free Cur had different fates in the intestinal tract. Both the concentration determinations and the fluorescence observations suggested that RA-Cur changed the biodistribution of Cur in the intestinal tract. Free Cur was uniformly distributed in the duodenum, jejunum, and ileum at the 0.5-hour time point, whereas the RA-Cur concentration in the duodenum was 3.16-fold that in the jejunum and 12.95-fold that in the ileum. The trend for Cur biodistribution in the intestinal tract at 1.5 hours was consistent with that at 0.5 hours for RA-Cur, with a rank order of duodenum $>>$ jejunum $>>$ ileum, while the rank order for Cur distribution of free Cur in different intestinal segments was ileum $>$ jejunum $>$ duodenum. Therefore, RA-Cur induced significant additional retention in the duodenum, indicating that RA-Cur could potentially target drugs to the duodenum in clinical applications.

However, the biodistribution of RA-Cur in the intestinal tract in the present study was only analyzed in terms of the cargo (Cur) embedded in the vehicle (RA micelles), and did not assess the vehicle directly. This study did not determine whether the entire RA-Cur micelle was taken up into the intestinal tissue or whether Cur was released first and then taken up into the tissues. The RA micelles were prone to disassembly and release of Cur after dilution upon entry into the gastrointestinal tract. Consequently, the biodistribution observed in the intestinal tract (Figure 5) could represent the localization of Cur and not the localization of the RA-Cur micelles. The fate of RA (non-assembled and/or assembled RA) in tissues was not evaluated in this study, as the main focus and the final aim were to explore novel nanomedicine techniques and to optimize of the fate of the Cur cargo.

Intestinal absorption mechanisms of nanoparticles have been studied in very few reports, ${ }^{21}$ so they are poorly understood. An absorption experiment using the everted intestinal ring model was performed here to attempt to reveal the mechanisms of interaction of RA-Cur with the intestinal tract. Results of the incubation experiments conducted at $4^{\circ} \mathrm{C}$ in the presence of $\mathrm{NaN}_{3}$ suggested that active endocytosis of RA-Cur was involved in intestinal absorption. Four endocytosis inhibitors that alter different endocytosis mechanisms resulted in the suppression of absorption to different extents, indicating that multiple transcytosis mechanisms, including caveolae-mediated, clathrin-mediated, caveolaeindependent, and clathrin-independent endocytosis and macropinocytosis, were involved in the absorption of RA-Cur (Figure S6). Nevertheless, the absorption of RA-Cur in the presence of $\mathrm{NaN}_{3}$ or at $4^{\circ} \mathrm{C}$ still occurred, indicating that other energy-independent pathways might also function in intestinal absorption. No inhibitor used in this test could completely block the absorption of RA-Cur, also indicating the complicated mechanisms involved in the internalization of RA-Cur by the intestinal tract.

The results from the pharmacokinetic studies were promising, as RA-Cur showed significantly higher relative bioavailability of Cur when compared to free Cur. The improved in vivo bioavailability of Cur could be attributed to the following: 1) RA micelle encapsulation enhanced the Cur solubility and dissolution rate in gastrointestinal fluids, thereby maintaining Cur molecules at high concentrations and accelerating Cur absorption; 2) RA micelles may protect Cur from degradation in the intestine, resulting in higher Cur levels in the blood circulation; 3) the ultrasmall size of RA micelles $(\sim 4 \mathrm{~nm})$ could facilitate mucus layer permeation, allowing the micelles to be absorbed by transcytosis mechanisms; and 4) the ultrasmall particle size of RA micelles also aided penetration through the paracellular pathway. ${ }^{42}$ In this respect, it is notable that the smallest sized particles are reported to have the highest cellular internalization via clathrin-mediated and caveolae-mediated endocytosis. ${ }^{43}$

The biodistribution and pharmacokinetic results for RACur indicated its anti-inflammatory potential in the intestinal tract. One of the two major inflammatory bowel diseases (IBD) of the gastrointestinal tract is UC, which is characterized by both acute and chronic inflammation of the intestine. ${ }^{44}$ The DSS colitis model is very popular in IBD research due to its rapid establishment, simplicity, reproducibility, and controllability, with intestinal inflammation achieved by an appropriate choice of DSS concentration and the frequency of administration. ${ }^{44}$ Several reports using a DSS-induced mouse $\mathrm{UC}$ model have revealed the therapeutic effectiveness of Cur in alleviating colitis. ${ }^{24,45,46}$ The present study now confirms the anti-inflammatory potential of RA-Cur in the intestinal tract.

The mice in the DSS control group had characteristics that included marked body weight loss, rectal bleeding, increased spleen weight, reduction in colon length, destruction of the intestinal epithelium, and massive infiltration of inflammatory cells. All these characteristics indicated successful establishment of the mouse UC model. Treatment with $150 \mathrm{mg} / \mathrm{kg}$ Cur for 6 days alleviated the body weight loss and spleen weight increases in the DSS-induced mice, and the colon length was rescued. Treatment with RA-Cur enabled dose-dependent protection against the symptoms of DSS colitis, as the effects of $150 \mathrm{mg} / \mathrm{kg}$ RA-Cur were more pronounced when compared to the response achieved with 50 and $100 \mathrm{mg} / \mathrm{kg} \mathrm{RA-Cur}$. These effects included a significantly alleviated body weight loss and spleen weight 
increase in response to DSS, and a rescued colon length. The results from histopathologic examinations also supported these findings.

The safeties of RA and Cur have been extensively evaluated, and the common perception is that both agents have a sufficiently long history and wide use to verify their oral safety. No contrary indications were noted regarding the safety of administration in any of the ex vivo/in vivo experiments in this work. Therefore, safety concerns regarding the oral use of RA-Cur were not addressed here.

The present work indicates a strong potential for extending the use of RA as an oral nanocarrier. RA micelles have many advantages, such as oral safety, low cost, high stability, high drug encapsulating capacity, and pronounced biological activity, when compared with other oral delivery systems. S. rebaudiana Bertoni is rich in RA and the extraction is simple with high yield. ${ }^{47}$ The fabrication process for the formulation of RA-Cur is simple and requires no hazardous substances, and the preparation protocol is easily adaptable to large-scale preparation if desired. Therefore, the RA-based self-nanomicellizing formulation meets the criteria for ecofriendly preparation. All the results of our study suggest that the RA-based self-nanomicellizing solid dispersion might be a promising nanocarrier system that can be used to improve the oral bioavailability of Cur and other hydrophobic drugs.

\section{Conclusion}

A novel self-nanomicellizing solid dispersion system based on RA was successfully prepared, and its physicochemical properties in solid state and solution were characterized. In vitro tests confirmed that RA-Cur significantly enhanced the apparent solubility, stability, simulated passive intestinal absorption, and antioxidant capacity of Cur, a hydrophobic drug. In vivo tests confirmed the biodistribution of RA-Cur and its endocytic uptake into the intestinal tract. In vivo studies also clearly demonstrated the significantly improved bioavailability and stronger anti-inflammation properties of RA-Cur when compared with free Cur. RA readily formed solid dispersions that dissolved and self-assembled into ultrasmall micelles $(\sim 4 \mathrm{~nm})$ in aqueous solutions. This RAbased self-nanomicellizing solid dispersion could therefore represent a promising green therapeutic tool for encapsulation of hydrophobic and unstable drugs such as Cur for the treatment of gastrointestinal tract disorders.

\section{Data availability}

All relevant data are within the paper and the Supplementary materials.

\section{Acknowledgments}

This research was supported by the National Natural Science Foundation of China (project nos 81770895 and 81500751) and the Project of Medical and Health Technology Development Program in Shandong Province, China (project no. 2017WS633).

\section{Author contributions}

All authors contributed to data analysis, drafting and revising the article, gave final approval of the version to be published, and agree to be accountable for all aspects of the work.

\section{Disclosure}

The authors report no conflicts of interest in this work.

\section{References}

1. Wang Y, Li L, Wang Y, et al. New application of the commercial sweetener rebaudioside a as a hepatoprotective candidate: Induction of the Nrf2 signaling pathway. Eur J Pharmacol. 2018;822:128-137.

2. Thøgersen R, Petrat-Melin B, Zamaratskaia G, Grevsen K, Young JF, Rasmussen MK. In vitro effects of rebaudioside A, stevioside and steviol on porcine cytochrome p450 expression and activity. Food Chem. 2018; 258:245-253.

3. Zhang M, Dai T, Feng N. A Novel Solubility-Enhanced RubusosideBased Micelles for Increased Cancer Therapy. Nanoscale Res Lett. 2017;12(1):274-258

4. Song K, Xin M, Yu H, et al. Novel ultra-small micelles based on rebaudioside A: A potential nanoplatform for ocular drug delivery. Int J Pharm. 2018;552(1-2):265-276.

5. Platania CBM, Fidilio A, Lazzara F, et al. Retinal Protection and Distribution of Curcumin in Vitro and in Vivo. Front Pharmacol. 2018;9:670.

6. Jamwal R. Bioavailable curcumin formulations: A review of pharmacokinetic studies in healthy volunteers. J Integr Med. 2018;16(6):367-374.

7. Zhang J, Chou G, Liu Z, Liu M. Employing rubusoside to improve the solubility and permeability of antitumor compound betulonic acid. Nanomedicine. 2016;29(14):2829-2844.

8. Wan ZL, Wang JM, Wang LY, Yang XQ, Yuan Y. Enhanced physical and oxidative stabilities of soy protein-based emulsions by incorporation of a water-soluble stevioside-resveratrol complex. J Agric Food Chem. 2013;61(18):4433-4440.

9. Uchiyama H, Tozuka Y, Nishikawa M, Takeuchi H. Nanocomposite formation between alpha-glucosyl stevia and surfactant improves the dissolution profile of poorly water-soluble drug. Int J Pharm. 2012; 428(1-2): 183-186.

10. Zhang F, Koh GY, Jeansonne DP, et al. A novel solubility-enhanced curcumin formulation showing stability and maintenance of anticancer activity. J Pharm Sci. 2011;100(7):2778-2789.

11. Zhang F, Koh GY, Hollingsworth J, Russo PS, Stout RW, Liu Z. Reformulation of etoposide with solubility-enhancing rubusoside. Int J Pharm. 2012;434(1-2):453-459.

12. Uchiyama H, Tozuka Y, Imono M, Takeuchi H. Transglycosylated stevia and hesperidin as pharmaceutical excipients: dramatic improvement in drug dissolution and bioavailability. Eur J Pharm Biopharm. 2010;76(2):238-244.

13. Wang H, Cui Y, Fu Q, et al. A phospholipid complex to improve the oral bioavailability of flavonoids. Drug Dev Ind Pharm. 2015;41(10): 1693-1703.

14. Guo C, Zhang Y, Yang Z, et al. Nanomicelle formulation for topical delivery of cyclosporine A into the cornea: in vitro mechanism and in vivo permeation evaluation. Sci Rep. 2015;5(1):12968. 
15. Guo C, Cui F, Li M, Li F, Wu X. Enhanced corneal permeation of coumarin-6 using nanoliposomes containing dipotassium glycyrrhizinate: in vitro mechanism and in vivo permeation evaluation. RSC Adv. 2015;5(92):75636-75647.

16. Xu T, Xu X, Gu Y, Fang L, Cao F. Functional intercalated nanocomposites with chitosan-glutathione-glycylsarcosine and layered double hydroxides for topical ocular drug delivery. Int $J$ Nanomedicine. 2018;13:917-937.

17. Kumar S, Kesharwani SS, Mathur H, Tyagi M, Bhat GJ, Tummala H Molecular complexation of curcumin with $\mathrm{pH}$ sensitive cationic copolymer enhances the aqueous solubility, stability and bioavailability of curcumin. Eur J Pharm Sci. 2016;82:86-96.

18. Yang R, Zhang S, Kong D, Gao X, Zhao Y, Wang Z. Biodegradable polymer-curcumin conjugate micelles enhance the loading and delivery of low-potency curcumin. Pharm Res. 2012;29(12):3512-3525.

19. Jahanshiri Z, Shams-Ghahfarokhi M, Allameh A, Razzaghi-Abyaneh M. Effect of Curcumin on Aspergillus parasiticus Growth and Expression of Major Genes Involved in the Early and Late Stages of Aflatoxin Biosynthesis. Iran J Public Health. 2012;41(6):72-79.

20. Ge W, Li D, Chen M, Wang X, Liu S, Sun R. Characterization and antioxidant activity of $\beta$-carotene loaded chitosan-graft-poly(lactide) nanomicelles. Carbohydr Polym. 2015;117:169-176.

21. Cheng B, Pan H, Liu D, et al. Functionalization of nanodiamond with vitamin E TPGS to facilitate oral absorption of curcumin. Int J Pharm. 2018;540(1-2):162-170.

22. Chen Q, Gou S, Huang Y, et al. Facile fabrication of bowl-shaped microparticles for oral curcumin delivery to ulcerative colitis tissue. Colloids Surf B Biointerfaces. 2018;169:92-98.

23. Zhang Q, Polyakov NE, Chistyachenko YS, et al. Preparation of curcumin self-micelle solid dispersion with enhanced bioavailability and cytotoxic activity by mechanochemistry. Drug Deliv. 2018;25(1): 198-209.

24. Xiao B, Si X, Zhang M, Merlin D. Oral administration of $\mathrm{pH}$-sensitive curcumin-loaded microparticles for ulcerative colitis therapy. Colloids Surf B Biointerfaces. 2015;135:379-385.

25. Lean QY, Eri RD, Fitton JH, Patel RP, Gueven N. Fucoidan Extracts Ameliorate Acute Colitis. PLoS One. 2015;10(6):e0128453.

26. Suzuki H, Ueno K, Mizumoto T, Seto Y, Sato H, Onoue S. Selfmicellizing solid dispersion of cyclosporine A for pulmonary delivery: Physicochemical, pharmacokinetic and safety assessments. Eur J Pharm Sci. 2017;96:107-114.

27. Onoue S, Suzuki H, Kojo Y, et al. Self-micellizing solid dispersion of cyclosporine A with improved dissolution and oral bioavailability. Eur J Pharm Sci. 2014;62:16-22.

28. Shi NQ, Zhang Y, Li Y, et al. Self-micellizing solid dispersions enhance the properties and therapeutic potential of fenofibrate: Advantages, profiles and mechanisms. Int J Pharm. 2017;528(1-2):563-577.

29. Li M, Xin M, Guo C, Lin G, Wu X. New nanomicelle curcumin formulation for ocular delivery: improved stability, solubility, and ocular anti-inflammatory treatment. Drug Dev Ind Pharm. 2017;43(11): 1846-1857.

30. Weuts I, Kempen D, Six K, et al. Evaluation of different calorimetric methods to determine the glass transition temperature and molecular mobility below T(g) for amorphous drugs. Int J Pharm. 2003;259(1-2) $17-25$.
31. Yu H, Huang Q. Enhanced in vitro anti-cancer activity of curcumin encapsulated in hydrophobically modified starch. Food Chem. 2010; 119(2):669-674.

32. Saravanan R, Ramachandran V. Modulating efficacy of Rebaudioside A, a diterpenoid on antioxidant and circulatory lipids in experimental diabetic rats. Environ Toxicol Pharmacol. 2013;36(2):472-483.

33. Kim KS, Suzuki K, Cho H, Youn YS, Bae YH. Oral Nanoparticles Exhibit Specific High-Efficiency Intestinal Uptake and Lymphatic Transport. ACS Nano. 2018;12(9):8893-8900.

34. Jung T, Kamm W, Breitenbach A, Kaiserling E, Xiao JX, Kissel T. Biodegradable nanoparticles for oral delivery of peptides: is there a role for polymers to affect mucosal uptake? Eur J Pharm Biopharm. 2000;50(1):147-160.

35. Agrawal U, Sharma R, Gupta M, Vyas SP. Is nanotechnology a boon for oral drug delivery? Drug Discov Today. 2014;19(10):1530-1546.

36. $\mathrm{Lu} \mathrm{Z}, \mathrm{Bu} \mathrm{C}, \mathrm{Hu} \mathrm{W}$, et al. Preparation and in vitro and in vivo evaluation of quercetin-loaded mixed micelles for oral delivery. Biosci Biotechnol Biochem. 2018;82(2):238-246.

37. Sun F, Jaspers TC, van Hasselt PM, Hennink WE, van Nostrum CF. A Mixed Micelle Formulation for Oral Delivery of Vitamin K. Pharm Res. 2016;33(9):2168-2179.

38. Feng B, Mills JB, Davidson RE, et al. In vitro P-glycoprotein assays to predict the in vivo interactions of P-glycoprotein with drugs in the central nervous system. Drug Metab Dispos. 2008;36(2):268-275.

39. Mensch J, Melis A, Mackie C, Verreck G, Brewster ME, Augustijns P. Evaluation of various PAMPA models to identify the most discriminating method for the prediction of BBB permeability. Eur J Pharm Biopharm. 2010;74(3):495-502.

40. Sun H, Nguyen K, Kerns E, et al. Highly predictive and interpretable models for PAMPA permeability. Bioorg Med Chem. 2017;25(3):1266-1276.

41. Avdeef A, Nielsen PE, Tsinman O. PAMPA - a drug absorption in vitro model 11. Matching the in vivo unstirred water layer thickness by individual-well stirring in microtitre plates. Eur J Pharm Sci. 2004; 22(5):365-374.

42. Pauletti GM, Okumu FW, Borchardt RT. Effect of size and charge on the passive diffusion of peptides across Caco- 2 cell monolayers via the paracellular pathway. Pharm Res. 1997;14(2):164-168.

43. Saw WS, Ujihara M, Chong WY, et al. Size-dependent effect of cystine/citric acid-capped confeito-like gold nanoparticles on cellular uptake and photothermal cancer therapy. Colloids Surf B Biointerfaces. 2018;161:365-374.

44. Chassaing B, Aitken JD, Malleshappa M, Vijay-Kumar M. Dextran (DSS)-induced colitis in mice. Curr Protoc Immunol. 2014; 104:Unit 15.25.

45. Kao NJ, Hu JY, Wu CS, Kong ZL. Curcumin represses the activity of inhibitor- $\mathrm{KB}$ kinase in dextran sulfate sodium-induced colitis by S-nitrosylation. Int Immunopharmacol. 2016;38:1-7.

46. Yang H, Du Z, Wang W, et al. Structure-Activity Relationship of Curcumin: Role of the Methoxy Group in Anti-inflammatory and Anticolitis Effects of Curcumin. J Agric Food Chem. 2017;65(22):4509-4515.

47. Ameer K, Bae SW, Jo Y, Lee HG, Ameer A, Kwon JH. Optimization of microwave-assisted extraction of total extract, stevioside and rebaudioside-A from Stevia rebaudiana (Bertoni) leaves, using response surface methodology (RSM) and artificial neural network (ANN) modelling. Food Chem. 2017;229:198-207.
International Journal of Nanomedicine

\section{Publish your work in this journal}

The International Journal of Nanomedicine is an international, peerreviewed journal focusing on the application of nanotechnology in diagnostics, therapeutics, and drug delivery systems throughout the biomedical field. This journal is indexed on PubMed Central, MedLine, CAS, SciSearch $®$, Current Contents $® /$ Clinical Medicine,

\section{Dovepress}

Journal Citation Reports/Science Edition, EMBase, Scopus and the Elsevier Bibliographic databases. The manuscript management system is completely online and includes a very quick and fair peer-review system, which is all easy to use. Visit http://www.dovepress.com/ testimonials.php to read real quotes from published authors. 